\title{
Photodetachment Near a Repulsive Center: Closed-Orbit Theory for the Total Cross Section
}

B. C. Yang

John B. Delos

William \& Mary, jbdelos@wm.edu

M. L. Du

Follow this and additional works at: https://scholarworks.wm.edu/aspubs

Part of the Physics Commons

\section{Recommended Citation}

Yang, B. C.; Delos, John B.; and Du, M. L., Photodetachment Near a Repulsive Center: Closed-Orbit Theory for the Total Cross Section (2014). Physical Review A, 89(1).

https://doi.org/10.1103/PhysRevA.89.013417

This Article is brought to you for free and open access by the Arts and Sciences at W\&M ScholarWorks. It has been accepted for inclusion in Arts \& Sciences Articles by an authorized administrator of W\&M ScholarWorks. For more information, please contact scholarworks@wm.edu. 


\title{
Photodetachment near a repulsive center: Closed-orbit theory for the total cross section
}

\author{
B. C. Yang, ${ }^{1}$ J. B. Delos, ${ }^{2}$ and M. L. Du ${ }^{1, *}$ \\ ${ }^{1}$ State Key Laboratory of Theoretical Physics, Institute of Theoretical Physics, Chinese Academy of Science, Beijing 100190, China \\ ${ }^{2}$ Physics Department, College of William and Mary, Williamsburg, Virginia 23185, USA
}

(Received 30 October 2013; published 21 January 2014)

\begin{abstract}
The total photodetachment cross section of a negative ion near a repulsive center is studied based on the closed-orbit theory. An analytical expression, written as a product of the zero-field photodetachment cross section and a modulation function, is obtained for energy above and below threshold. The expression also incorporates the different wave sources produced by different negative ions. The present results are shown to be accurate by comparing with quantum calculations based on the exact Coulomb Green's function. We also compared the photodetachment cross section near a repulsive center with that in a homogeneous electric field and found interesting connections between the two.
\end{abstract}

DOI: 10.1103/PhysRevA.89.013417

PACS number(s): 32.80.Gc, 31.15.xg

\section{INTRODUCTION}

Recently, we examined a theoretical model of photodetachment near a repulsive center [1]. This work was inspired by an experiment on the photodetachment of a dicarboxylate dianion [2], ${ }^{-} \mathrm{O}_{2} \mathrm{C}-\left(\mathrm{CH}_{2}\right)_{m}-\mathrm{CO}_{2}^{-}$(with $3 \leqslant m \leqslant 11$ ), in which the photoelectron spatial distribution was found to be largely dependent on the intramolecular Coulomb repulsive force. In the previous paper [1], we discussed the quantum interference in the differential cross sections induced by the nearby repulsive center. Here we consider the effects of the nearby repulsive center on the total photodetachment cross section.

Many theoretical studies have revealed the phenomena induced by external fields in the photodetachment spectra of negative ions [3-11] (see also references in [1]). Three dominant phenomena stand out in these studies: oscillatory structures above the zero-field threshold, a finite cross-section value at the threshold, and a quantum tunneling effect below threshold. These phenomena have been observed in some experiments [12-14]. In addition to these, several approaches $[3,4,7,8]$ have explored the final-state interaction between the detached electron and the remaining neutral atom after photodetachment. A related quantum-scattering problem of slow electrons by a zero-range potential and a Coulomb potential has been discussed by Rabinovich in 1985 [15].

In this paper, we use closed-orbit theory $[6,16]$ to investigate the effects of a nearby repulsive center on the total photodetachment cross section of a negative ion. The theory provides a clear physical picture for the oscillations in the total cross sections. The present results are shown to be accurate by comparing with quantum calculations based on the exact Coulomb Green's function.

The remainder of this article is organized as follows. After briefly summarizing the theoretical model and the electron wave propagation after detachment in Sec. II, we first use the closed-orbit theory to obtain a formula for the oscillatory structure in the photodetachment spectra above the zero-field threshold. Then we extend it to energy below the zero-field threshold by introducing a uniform approximation in Sec. III.

\footnotetext{
*duml@itp.ac.cn
}

Several interesting phenomena are discussed in Sec. IV and a comparison is made with the photodetachment of a negative ion in a homogeneous electric field. Finally, a conclusion is given in Sec. V. Atomic units are used throughout this work unless specified otherwise.

\section{THEORETICAL MODEL AND PHOTOELECTRON WAVE PROPAGATION}

The picture of photodetachment near a repulsive center (Fig. 1) is identical to that in the previous work [1]. However, previously we found it was convenient to study the differential cross sections using a coordinate system $(r, \theta)$ with its origin at the repulsive center (solid circle). Here, we find that it is more convenient to use a coordinate system with its origin at the source point (open circle), because we have to follow a closed orbit going out from and returning to the source, and we also evaluate overlap integrals of wave functions relative to the source point. We use the subscript $s$ to indicate coordinates $\left(r_{s}, \theta_{s}\right)$ relative to the source (Fig. 1).

The theoretical model for the photodetachment of negative ions is well established [3-11,13]. Initially an electron is loosely bound by a short-range potential $V\left(r_{s}\right)$ in a state $\psi_{i}$. After absorbing a photon, the loosely bound electron is detached and an outgoing electron wave $\psi_{\text {out }}$ is generated from the detaching region. The outgoing wave is related to the initial state $\psi_{i}$ by the following inhomogeneous Schrödinger equation:

$$
\frac{1}{2}\left(\nabla_{s}^{2}+k^{2}-2 V\right) \psi_{\text {out }}=D \psi_{i},
$$

where $D$ is a dipole operator and $k=\sqrt{2 E}$, with $E$ denoting the initial kinetic energy. When the distance $r_{s}$ is sufficiently large $\left(k r_{s} \gg 1\right)$, the detached-electron wave behaves as a spherical outgoing wave because the short-range potential $V$ is vanishingly small.

After detachment from the loosely bound initial state and away from the source region, the detached-electron motion is governed by the Hamiltonian near a repulsive center:

$$
H=\frac{p_{r}^{2}}{2}+\frac{p_{\theta}^{2}}{2 r^{2}}+\frac{\alpha}{r}-\frac{\alpha}{d},
$$

where $d$ is the distance between the wave source and the repulsive center and $\alpha$ denotes the negative-charge number 


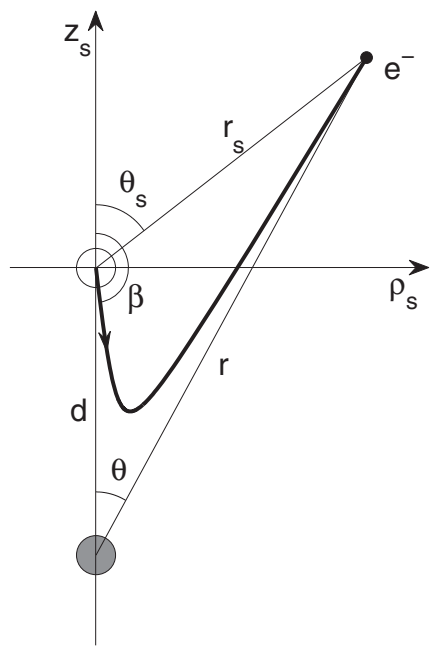

FIG. 1. Schematic representation of the system. The open and the solid circles denote an electron source and a negatively charged center, respectively. The electron source is supplied by photodetachment of electrons from a negative ion. After the electron is detached, it escapes following a hyperbolic trajectory as shown by the heavy solid curve. Two coordinate frames are displayed with one relative to the repulsive center and the other relative to the source of the electron. The angle $\beta$ is the initial ejection angle of the detached electron relative to the $z$ axis.

at the force center. Note the last term $\frac{\alpha}{d}$ is a constant and its purpose is to shift the value of the repulsive potential at the position of the negative ion to zero. The classical trajectory analysis for the present model has been presented in our previous work [1], from which one can immediately find a classical closed orbit lying on the line between the electron source and the repulsive center (see Fig. 2 in [1]). A part of the outgoing wave follows the closed orbit and comes back to the source region.

In order to obtain the returning wave function, we first select an initial spherical surface of radius $R_{S}$ centered at the negative ion $[6,16,17]$. The radius is such that on the spherical surface the detached-electron outgoing wave is asymptotic and the external repulsive force can be safely neglected compared with the kinetic energy of the detached electron. This requires

$$
\frac{1}{k} \ll R_{s} \ll \frac{\widetilde{E}}{\widetilde{E}+1} d,
$$

where $\widetilde{E}=\frac{E d}{\alpha}$ is a scaled energy [1]. Such $R_{s}$ exists as long as $\frac{1}{k} \ll \frac{\widetilde{E}}{\tilde{E}+1} d$ or the energy is not very close to the zero-field threshold. The final physical result is independent of such a surface radius $R_{S}$ as shown in the following sections.

On the spherical surface we assume the detached-electron wave has the following spherical outgoing-wave form:

$$
\psi_{\text {out }}\left(R_{s}, \beta, \phi\right)=C(k) Y_{l m}(\beta, \phi) \frac{e^{i k R_{s}}}{R_{s}},
$$

where $\left(R_{s}, \beta, \phi\right)$ are spherical coordinates for the electron relative to the source point (Fig. 1); $C(k)$ is a factor dependent on the electron energy $E$. The angle-dependent factor $Y_{l m}(\beta, \phi)$ is a spherical harmonic function representing the angular distribution of outgoing waves. Usually we are interested in

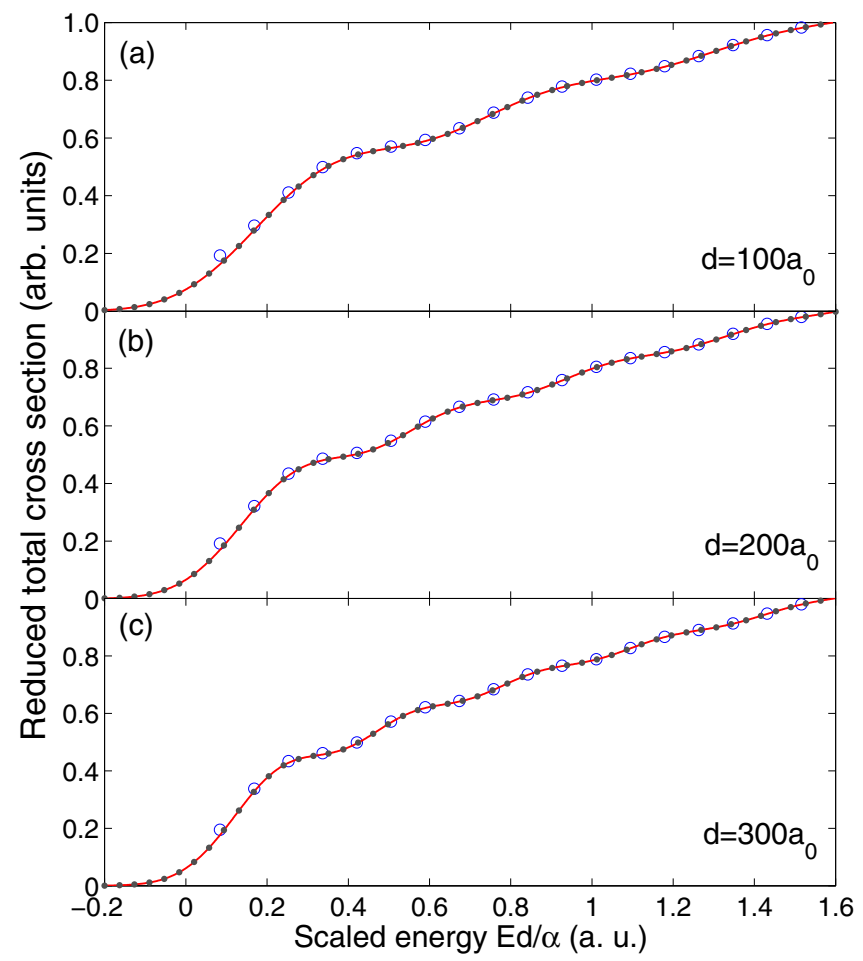

FIG. 2. (Color online) Reduced total cross sections in Eq. (22) for an $s$-wave source (solid curves) with $\alpha=1$ and (a) $d=100 a_{0}$, (b) $d=200 a_{0}$, and (c) $d=300 a_{0}$, where $a_{0}$ is the Bohr radius. The open circles are results obtained by numerically integrating the detachedelectron flux distributions [1]. The dots represent the quantum results using the formulas in Appendix D.

an $s$-wave source, such as the photodetachment of $\mathrm{S}^{-}[13,14]$, or a $p$-wave source such as the photodetachment of $\mathrm{H}^{-}$[12].

The semiclassical propagation of such an initial spherical outgoing wave in Eq. (4) has been analyzed in detail earlier [1] . The general semiclassical wave propagating along the closed orbit can be written as

$$
\psi_{\text {ret }}=\psi_{\text {out }}\left(R_{S}, \pi, \phi\right) A(\mathbf{r}) e^{i\left[S(\mathbf{r})-\mu \frac{\pi}{2}\right]},
$$

where $\mu$ is the Maslov index, $S(\mathbf{r})$ is the classical action, and $A(\mathbf{r})$ is the amplitude. When the wave comes back to the source, the Maslov index equals 1, and the semiclassical amplitude $A$ and the accumulated phase $S$ are, respectively, given by

$$
A=\frac{\alpha R_{s}}{4 E d^{2}}
$$

and

$$
\begin{aligned}
S & =\frac{2 \sqrt{2 d \alpha}}{\sqrt{1+\widetilde{E}}}[\sqrt{\widetilde{E}(1+\widetilde{E})}-\ln (\sqrt{1+\widetilde{E}}+\sqrt{\widetilde{E}})], \\
\widetilde{E} & \geqslant 0
\end{aligned}
$$

The amplitude $A$ in Eq. (6) is obtained from Eqs. (23) and (26) in [1], and the phase $S$ in Eq. (7) is obtained from Eq. (67) in [1]. The action $S$ along this closed orbit can also be calculated using 
the standard definition

$$
S=2 \int_{r_{\mathrm{turn}}}^{d} \sqrt{2\left(E+\frac{\alpha}{d}-\frac{\alpha}{r}\right)} d r
$$

with the turning point $r_{\text {turn }}=\frac{\alpha}{E+\alpha / d}$, by assuming $\sqrt{r}=x$ and integrating by parts.

When the detached-electron wave in Eq. (5) returns to the source region, the returning wave in Eq. (5) is approximately a plane wave traveling along the positive $z$ direction [6], and it can be written as

$$
\psi_{\mathrm{ret}}^{s}=\left[\frac{C(k) Y_{l m}(\pi, \phi)}{R_{s}}\right] A e^{i\left(S-\frac{\pi}{2}\right)} e^{i k z_{s}},
$$

where [18]

$$
Y_{l m}(\pi, \phi)=(-1)^{l} N_{l 0} \delta_{m 0}, \quad \text { with } \quad N_{l 0}=\sqrt{\frac{2 l+1}{4 \pi}} .
$$

We note that Eq. (9) is independent of $R_{S}$ because of the amplitude $A$ in Eq. (6). The returning wave in Eq. (9) overlaps with the quantum wave source and induces oscillatory structures in the total photodetachment cross section, which are described quantitatively in the next section.

\section{PHOTODETACHMENT CROSS SECTION}

In this section, we first give the essential formulas of closedorbit theory and then express the cross section in terms of only the outgoing wave and the returning wave that were given in Sec. II. A two-term expression is subsequently obtained for the total cross section above zero-field threshold. The formula is extended using a uniform approximation, which is applicable for all energy including the tunneling region below the zerofield threshold.

\section{A. Closed-orbit theory}

Following closed-orbit theory $[6,16]$, the total cross section of photodetachment in an external field can be written as

$$
\sigma=\sigma_{0}+\sigma_{r},
$$

where $\sigma_{0}$ is a smooth background

$$
\sigma_{0}=-\frac{4 \pi E_{\mathrm{ph}}}{c} \operatorname{Im}\left\langle D \psi_{i} \mid \psi_{\text {out }}\right\rangle
$$

corresponding to the photoabsorption process without any external field, and

$$
\sigma_{r}=-\frac{4 \pi E_{\mathrm{ph}}}{c} \operatorname{Im}\left\langle D \psi_{i} \mid \psi_{\mathrm{ret}}^{s}\right\rangle
$$

is the contribution from returning waves associated with closed orbits in an external field; $c$ and $E_{\mathrm{ph}}=E+E_{b}$ are, respectively, the light speed and the absorbed photon energy, with $E_{b}=k_{b}^{2} / 2$ denoting the binding energy of the negative ion.

Using the relationship between the detached-electron outgoing wave and an initial bound state in Eq. (1), the imaginary parts in Eqs. (12) and (13) can be, respectively, transformed into (Appendix A)

$$
\operatorname{Im}\left\langle D \psi_{i} \mid \psi_{\text {out }}\right\rangle=-\frac{1}{2} \operatorname{Im} \int\left(\psi_{\text {out }}^{*} \nabla_{r_{s}} \psi_{\text {out }}\right) d s_{R}
$$

for the direct-outgoing wave $\psi_{\text {out }}$ and

$$
\operatorname{Im}\left\langle D \psi_{i} \mid \psi_{\text {ret }}^{s}\right\rangle=\frac{1}{2} \operatorname{Im} \int\left(\psi_{\text {out }} \nabla_{r_{s}} \psi_{\text {ret }}^{s^{*}}-\psi_{\text {ret }}^{s^{*}} \nabla_{r_{s}} \psi_{\text {out }}\right) d s_{R}
$$

for the returning wave $\psi_{\text {ret }}^{s}$, where $s_{R}$ is an area element on the spherical surface with a radius $R_{s}$ centered at the negative ion.

\section{B. Modulations above zero-field threshold induced by closed orbit}

Substituting the outgoing wave in Eq. (4) into Eq. (14), the smooth background without any external field can be immediately worked out as

$$
\sigma_{0}=\frac{2 \pi k E_{\mathrm{ph}}}{c}|C(k)|^{2} .
$$

Substituting the outgoing wave in Eq. (4) and the returning wave in Eq. (9) into Eq. (15), the integrals can be worked out as (Appendix B)

$$
\begin{aligned}
\int \psi_{\mathrm{out}} \nabla_{r_{s}} \psi_{\mathrm{ret}}^{s^{*}} d s_{R \rightarrow \infty}= & (-1)^{l}(2 l+1)|C(k)|^{2} \delta_{m 0} \frac{A}{2 R_{s}} \\
& \times\left[1+e^{i\left(2 k r_{s}-l \pi\right)}\right] e^{-i\left(S-\frac{\pi}{2}\right)}
\end{aligned}
$$

and

$$
\begin{aligned}
\int \psi_{\text {ret }}^{s^{*}} \nabla_{r_{s}} \psi_{\text {out }} d s_{R \rightarrow \infty}= & -(-1)^{l}(2 l+1)|C(k)|^{2} \delta_{m 0} \frac{A}{2 R_{s}} \\
& \times\left[1-e^{i\left(2 k r_{s}-l \pi\right)}\right] e^{-i\left(S-\frac{\pi}{2}\right)}
\end{aligned}
$$

Therefore, the oscillatory part associated with the closed orbit is

$$
\sigma_{r}=-(-1)^{l}(2 l+1)|C(k)|^{2} \frac{2 \pi A E_{\mathrm{ph}}}{c R_{s}} \cos (S) \delta_{m 0} .
$$

Finally, by combining the smooth background in Eq. (16) and the oscillatory part in Eq. (19), the total photodetachment cross section can be obtained as a product:

$$
\sigma=\sigma_{0} \mathcal{H}^{\mathbf{c}}
$$

where

$$
\mathcal{H}^{\mathbf{c}}=1-(-1)^{l}(2 l+1) \frac{A}{k R_{s}} \cos (S) \delta_{m 0}
$$

is the modulation function induced by returning waves along the closed orbit in the repulsive Coulomb field.

The total photodetachment cross section in Eq. (16) without any external fields must follow a Wigner power law [19,20], $\sigma_{0} \propto k^{2 l+1}$, near the zero-field threshold, which requires $C(k) \propto k^{l}$. For convenience in the discussions later, a reduced cross section can be defined as

$$
\widetilde{\sigma}=k^{2 l+1}\left(\frac{\sigma}{\sigma_{0}}\right)=k^{2 l+1} \mathcal{H}^{\mathbf{c}},
$$

which avoids the divergence of the modulation function $\mathcal{H}^{\mathbf{c}}$ at the zero-field threshold and also converges to the Wigner threshold law when the external field is removed. 


\section{Extending to the below threshold region}

Very close to the zero-field threshold, the semiclassical cross section obtained in Eqs. (20)-(22) is not accurate. For instance, for an $s$-wave source, it diverges at the zero-field threshold. Here we use a uniform approximation and extend the formulas to regions including threshold and below threshold.

We first rewrite the modulation function in Eq. (21) as

$$
\begin{aligned}
\mathcal{H}^{\mathbf{c}}= & {\left[1-(-1)^{l}(2 l+1) \frac{1}{3 S} \cos (S) \delta_{m 0}\right] } \\
& -(-1)^{l}(2 l+1)\left(\frac{A}{k R_{S}}-\frac{1}{3 S}\right) \cos (S) \delta_{m 0},
\end{aligned}
$$

where the first term in the square bracket is the semiclassical modulation function in a static electric field. When the zerofield threshold is approached, the first term dominates due to the following limit:

$$
\lim _{E \rightarrow 0}\left[\frac{A}{k R_{S}} / \frac{1}{3 S}\right]=1
$$

with the amplitude in Eq. (6) and the action in Eq. (7). Close to the zero-field threshold, the detached electron will be mainly affected by the repulsive potential very close to the wavesource location. Accordingly, the modulation function should be similar to that in a uniform electric field (Appendix C). For photodetachment in a uniform electric field, it is fortunate that the exact modulation function is available $[4,9,10]$.

We now replace the expression in the square bracket of Eq. (23) by an exact modulation function $\mathcal{H}^{F}$ in a uniform-field case corresponding to action $S$, and we replace the second term in Eq. (23) by more accurate Airy functions [1]. Then the modulation function in Eq. (23) becomes

$$
\begin{aligned}
\mathcal{H}^{\mathbf{c}}= & \mathcal{H}^{F}(\zeta)+(-1)^{l}(2 l+1)\left(\frac{A}{k R_{S}}-\frac{1}{3 S}\right) \\
& \times 2 \pi \operatorname{Ai}(\zeta) \operatorname{Ai}^{\prime}(\zeta) \delta_{m 0},
\end{aligned}
$$

where $\zeta$ is a real variable defined as

$$
\zeta=-\left(\frac{3}{4} S\right)^{2 / 3}
$$

and the function $\mathcal{H}^{F}$ can be written out as

$$
\mathcal{H}_{s}^{F}(\zeta)=\frac{\pi}{(-\zeta)^{\frac{1}{2}}}\left[\mathrm{Ai}^{\prime 2}(\zeta)-\zeta \operatorname{Ai}^{2}(\zeta)\right]
$$

for an $s$-wave source $(l=0, m=0)$ and as

$$
\mathcal{H}_{p_{z}}^{F}(\zeta)=\frac{\pi}{(-\zeta)^{\frac{3}{2}}}\left[\zeta^{2} \mathrm{Ai}^{2}(\zeta)-2 \mathrm{Ai}(\zeta) \mathrm{Ai}^{\prime}(\zeta)-\zeta \mathrm{Ai}^{\prime 2}(\zeta)\right]
$$

for a $p$-wave source $(l=1, m=0)$. The general form of the function $\mathcal{H}^{F}$ is given in Appendix $\mathrm{C}$.

The propagation amplitude $A$ in Eq. (6) can be directly evaluated in the negative electron-energy range. Both the electron momentum $k$ and the semiclassical-propagation phase $S$ can be extended from their definitions [see Eq. (8) for the original definition of $S$ ]. For energy below the zero-field threshold,

$$
k=i \sqrt{2(-E)}, \quad E \leqslant 0
$$

and

$$
S=-2 i \int_{d}^{r_{\text {turn }}} \sqrt{2\left(\frac{\alpha}{r}-\frac{\alpha}{d}-E\right)} d r, \quad E \leqslant 0,
$$

which can be worked out as

$$
\begin{aligned}
S & =\frac{2 i \sqrt{2 d \alpha}}{\sqrt{1+\widetilde{E}}}\left[\sqrt{-\widetilde{E}(1+\widetilde{E})}-\frac{1}{2} \arccos (1+2 \widetilde{E})\right] \\
-1 & <\widetilde{E} \leqslant 0
\end{aligned}
$$

and

$$
S=-i \infty, \quad \widetilde{E} \leqslant-1
$$

\section{CALCULATIONS AND DISCUSSIONS}

In this section, we first discuss several important general predictions derived from formulas in the last section. Then the photodetachment of hydrogen negative ion near a repulsive center is discussed in detail. Finally, a comparison is made between the photodetachment spectra near a repulsive center and that in a homogeneous electric field.

\section{A. Oscillations in the photodetachment cross section}

In Figs. 2 and 3 the reduced cross sections in Eq. (22) are plotted using Eqs. (25)-(32) for an $s$-wave source $(l=0$, $m=0)$ and a $p$-wave source $(l=1, m=0)$, respectively, where the oscillatory "ripple" structure can be observed clearly. With increasing distance between the electron source and the repulsive center, the oscillation frequency increases but the amplitude of the oscillation decreases.

We have also calculated the cross sections by numerically integrating the uniform differential cross section studied earlier [1]. The results of the numerical integrations are displayed as open circles in both Figs. 2 and 3. The agreements above the zero-field threshold are confirmed. Alternative calculations indicated by dots based on the exact Coulomb Green's function (Appendix D) further confirm the accuracy of the present results, even below the zero-field threshold.

For a detached-electron wave with $m \neq 0$, no oscillatory structure is predicted from the expression in Sec. III. In fact, the closed orbit sits exactly on the node of the electron outgoing wave and the oscillation is extremely weak [5,21]. A more refined treatment is required, which is a topic of future study.

\section{B. Shift of the photoelectron threshold}

In both Figs. 2 and 3 the photodetachment cross sections below the zero-field threshold are still significant because of quantum tunneling through the Coulomb barrier. According to the modulation function in Eq. (25), the photodetachment cross section vanishes once the scaled energy $\widetilde{E}$ approaches -1 . Therefore, as a result of the presence of the nearby repulsive center, the photoelectron threshold is shifted to a lower value equal to $E_{b}-\alpha / d$ from the zero-field photodetachment threshold $E_{b}$. This lowering of the photoelectron threshold has been observed experimentally [2,22]. 


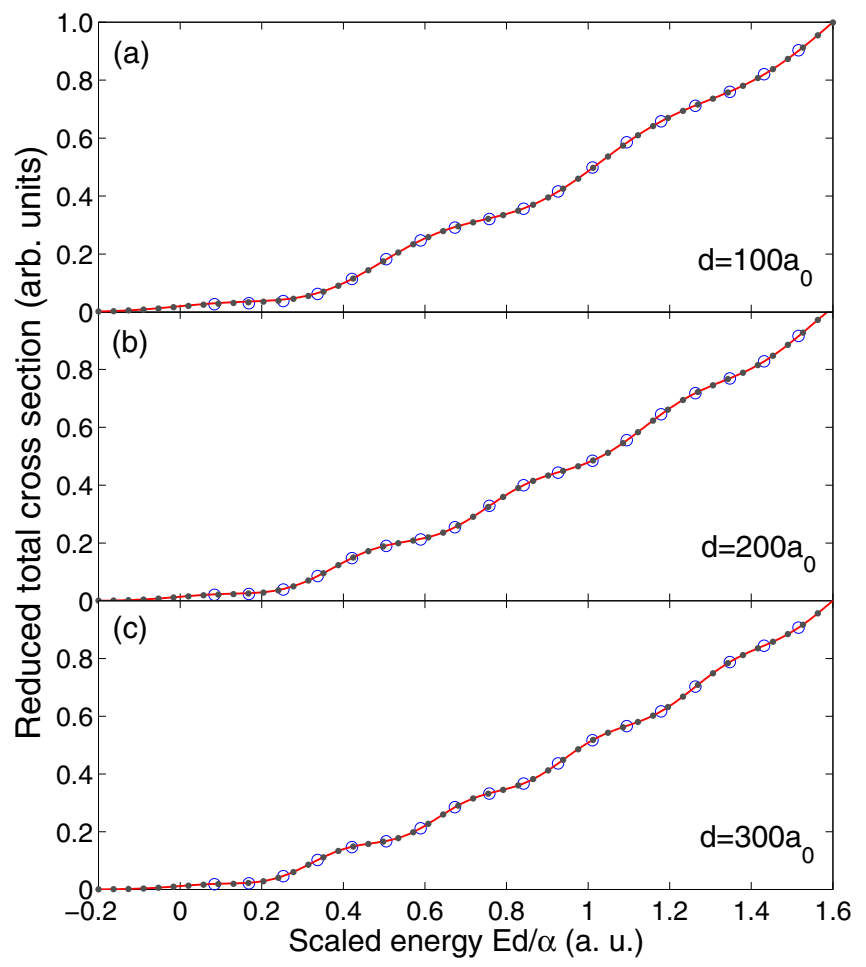

FIG. 3. (Color online) The same as Fig. 2 but for a $p_{z}$-wave source.

\section{Phase and scaling in modulation function}

The oscillatory phase in the photodetachment cross section is dependent on the symmetry of the quantum wave source, due to the existence of the parity factor $(-1)^{l}$ in the modulation function. From Eqs. (20) and (21), the parity factor $(-1)^{l}$ in the cross section can change the modulation phase as

$$
(-1)^{l} \cos (S)=\cos (S+l \pi) \text {. }
$$

In Fig. 4 we compare the modulation functions $\mathcal{H}^{\mathbf{c}}$ for an $s$-wave source and a $p_{z}$-wave source. A phase difference of about $\pi$ can be observed clearly. Such a source-dependent phase effect has been noticed earlier in studying the difference between the photodetachment spectra for an $s$-wave source and a $p$-wave source in a uniform electric field [23].

Next, we note that the scaling in the modulation function is similar to that in the differential cross section [1]. Noting that the action $S$ is a function only of the scaled energy $\widetilde{E}$ and the product of $d$ and $\alpha$, and also that

$$
\frac{A}{k R_{s}}=\frac{1}{4 \widetilde{E}^{\frac{3}{2}}} \frac{1}{\sqrt{2 d \alpha}},
$$

one can immediately verify that the modulation function $\mathcal{H}^{\mathbf{c}}$ in Eq. (25) is only dependent on the scaled energy $\widetilde{E}$ and the product $d \alpha$.

\section{Photodetachment of $\mathrm{H}^{-}$near a repulsive center}

Here, we consider the photodetachment spectra of $\mathrm{H}^{-}$ in more detail. The photodetachment of $\mathrm{H}^{-}$has been well established theoretically in the literature and the specific form of the spherical outgoing wave in Eq. (4) can be obtained from

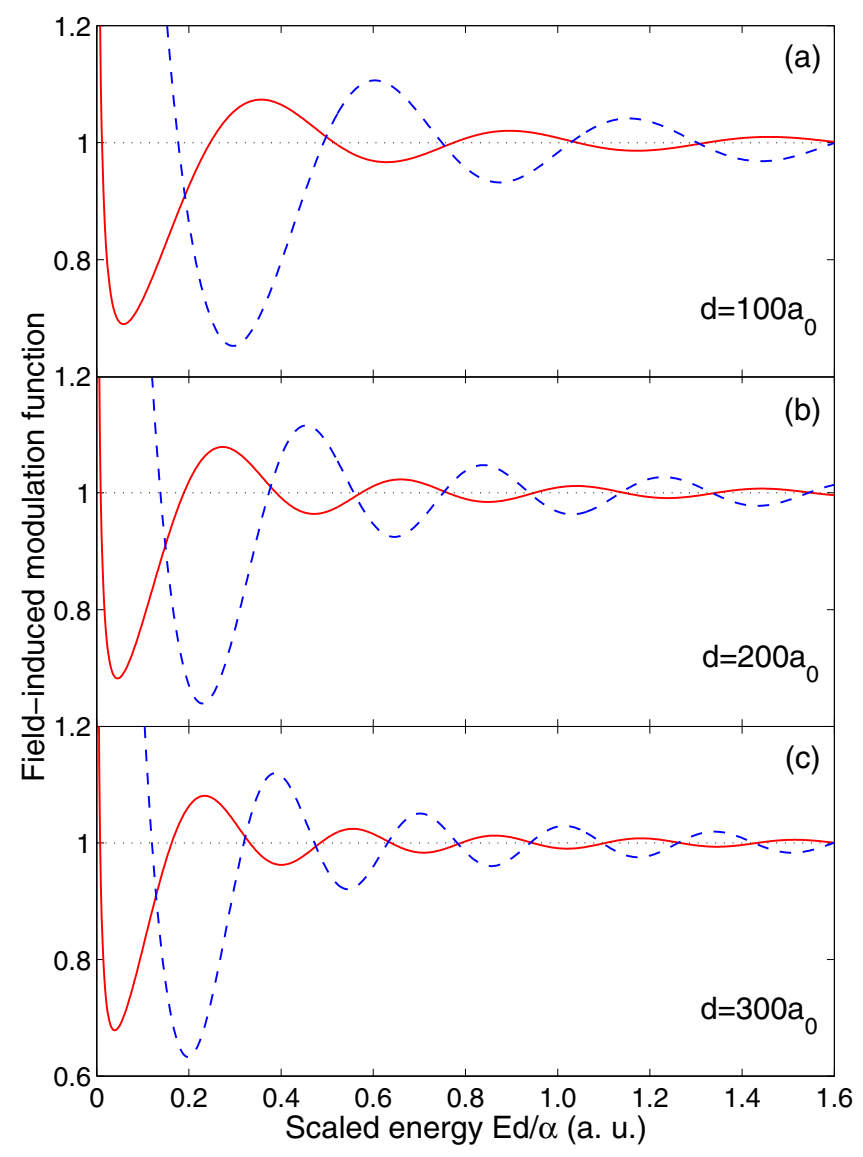

FIG. 4. (Color online) The modulation functions in Eq. (25) corresponding to the cases in Figs. 2 and 3. The solid curves are for an $s$-wave source, while the dashed curves are for a $p_{z}$-wave source. Note the phase difference of $\pi$ between the $s$-wave source and the $p_{z}$-wave source.

its initial bound state, which enables us to write out the total photodetachment cross section completely.

In the photodetachment process of $\mathrm{H}^{-}$irradiated by a linearly polarized laser light along the $z$ direction, from Eq. (1) the outgoing wave can be obtained as [17]

$$
\psi_{\mathrm{out}}=\frac{4 B k i}{\left(k_{b}^{2}+k^{2}\right)^{2}} \frac{e^{i k R_{s}}}{R_{s}} \cos \beta,
$$

where $B=0.31552$ is a normalization constant related to the initial bound state and the binding energy $E_{b}=0.7542 \mathrm{eV}$. By comparing with Eq. (4), the energy-dependent factor $C(k)$ has the following form:

$$
C(k)=\frac{i k B}{N_{10} E_{\mathrm{ph}}^{2}} .
$$

By combining Eqs. (16) and (21), the closed-orbit theory gives the total photodetachment cross section of $\mathrm{H}^{-}$near a repulsive center as

$$
\sigma=\frac{16 \pi^{2} \sqrt{2} B^{2} E^{3 / 2}}{3 c\left(E_{b}+E\right)^{3}}+\frac{4 \pi^{2} B^{2}}{c\left(E_{b}+E\right)^{3}} \frac{\alpha}{d^{2}} \cos (S), \quad \widetilde{E}>0 .
$$




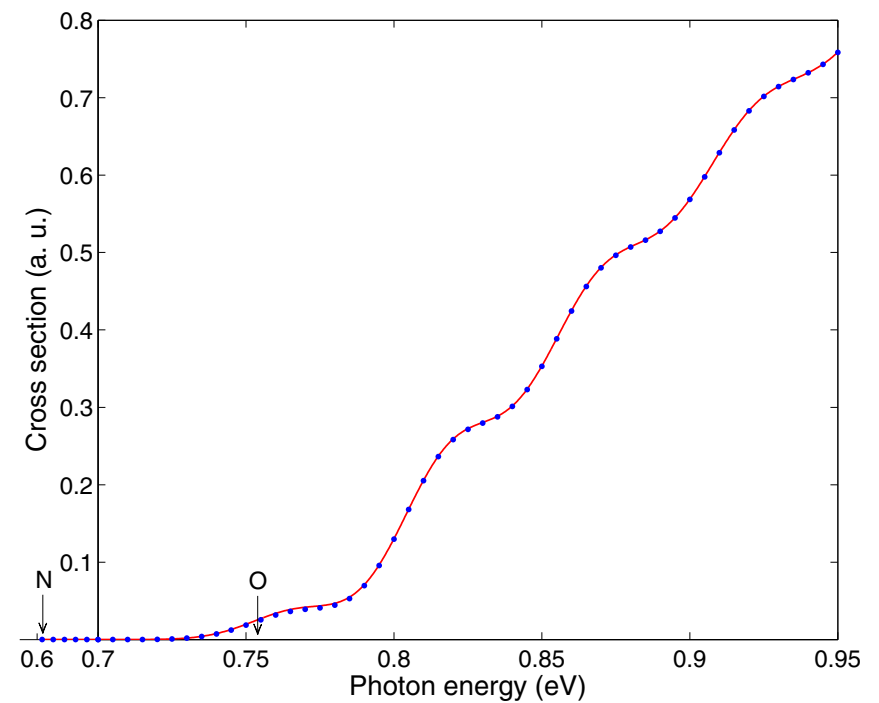

FIG. 5. (Color online) Photodetachment cross section of $\mathrm{H}^{-}$near a repulsive center in Eq. (38) with $d=200 a_{0}$ and $\alpha=1$. The accuracy is confirmed by an alternative calculation (dots) based on the exact Coulomb Green's function (Appendix D). Because of the presence of the repulsive center, the zero-field threshold denoted by the arrow $\mathrm{O}$ is shifted to a lower energy indicated by the arrow $\mathrm{N}$. Note that the energy scale below $0.7 \mathrm{eV}$ is different from that above $0.7 \mathrm{eV}$.

The uniform expression for the photodetachment cross section valid below and above the zero-field threshold is obtained by the product of the zero-field cross section or the first term on the right-hand side in the above equation and the modulation function in Eqs. (25) and (28). It is

$$
\begin{aligned}
\sigma= & \frac{16 \pi^{2} \sqrt{2} B^{2} E^{3 / 2}}{3 c\left(E_{b}+E\right)^{3}} \frac{\pi}{(-\zeta)^{\frac{3}{2}}}\left\{\zeta^{2} \mathrm{Ai}^{2}(\zeta)\right. \\
& \left.-\frac{1}{2}\left[1+\frac{6 \alpha}{k^{3} d^{2}}(-\zeta)^{\frac{3}{2}}\right] \operatorname{Ai}(\zeta) \operatorname{Ai}^{\prime}(\zeta)-\zeta \operatorname{Ai}^{\prime 2}(\zeta)\right\}
\end{aligned}
$$

with $\zeta$ given in Eq. (26).

In Fig. 5, a typical photodetachment cross section for $\mathrm{H}^{-}$near a repulsive center is displayed with $d=200 a_{0}$ and $\alpha=1$, where both the oscillatory structure above the zero-field threshold and the quantum tunneling effect below threshold can be observed clearly. The quantum calculations using Eq. (D7) from Appendix D is also shown, confirming the accuracy of the present uniform expression in Eq. (38).

\section{E. Comparing with the photodetachment in a homogeneous electric field}

When the photodetachment cross section near a repulsive center in Eq. (37) is compared with that in a uniform electric field [5,6], we find that the oscillatory amplitude in the photodetachment cross section near a repulsive center is the same as that in a uniform static field with field strength $F=\alpha / d^{2}$. In Fig. 6 we compare the cross section in Fig. 5 with the total photodetachment cross section of $\mathrm{H}^{-}$in a uniform static field with $F=128.55 \mathrm{kV} / \mathrm{cm}$, which is calculated from $F=\frac{\alpha}{d^{2}}$ in a.u. with $d=200 a_{0}$ and $\alpha=1$. In the figure

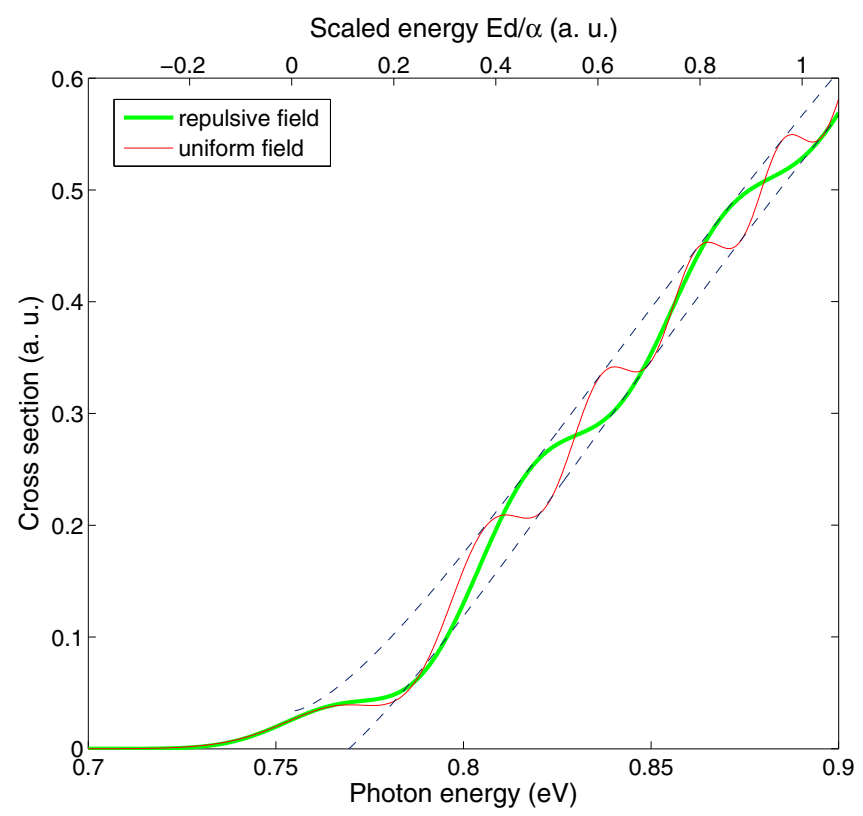

FIG. 6. (Color online) Comparing the photodetachment cross section of $\mathrm{H}^{-}$near a repulsive center (green and thick) with that in a uniform electric field (red and thin). The dashed lines represent the envelopes of the oscillations. Here $d=200 a_{0}$ and $\alpha=1$. The static electric-field strength is calculated from $F=\frac{\alpha}{d^{2}}$ and is equal to $128.55 \mathrm{kV} / \mathrm{cm}$. Note that the oscillations in the two systems have equal amplitudes, as discussed in the text.

the dashed lines are the envelopes of the oscillations. This equivalence may come as a surprise because $F=\alpha / d^{2}$ is only a local approximation to the repulsive potential in the negative ion region and the differential cross sections of the two systems seem very different $[1,17]$.

The origin of this equivalence can be traced to the dynamics of the two systems. In Fig. 7 we show trajectories near the closed orbits in both systems propagating out from and returning back to the negative ion. The thin lines (red online) are for a repulsive Coulomb field, while those thick lines (green online) are for the corresponding homogenous electric field. The dashed curve indicates the crossing points of the two trajectories in the two systems initially going out from the negative ion with the same ejection angle. Near the $z$ axis, the dashed line is a straight line crossing the origin where the negative ion is located and is perpendicular to the $z$ axis. The wave amplitude of the detached electron is related to the spacing of trajectories [1]. From Fig. 7, we note that, although the spacing of trajectories along the $z$ axis in each system varies as the position changes, nevertheless, two trajectories with the same outgoing angle in the two systems cross again on the dashed line, leading to the same spacing of trajectories and returning wave amplitude. Because of the dynamical origin, the discussion above about the negative hydrogen ion is equally valid for other negative ions.

\section{CONCLUSIONS}

Following our recent study on the quantum interferences in the differential cross section [1], we have studied the 


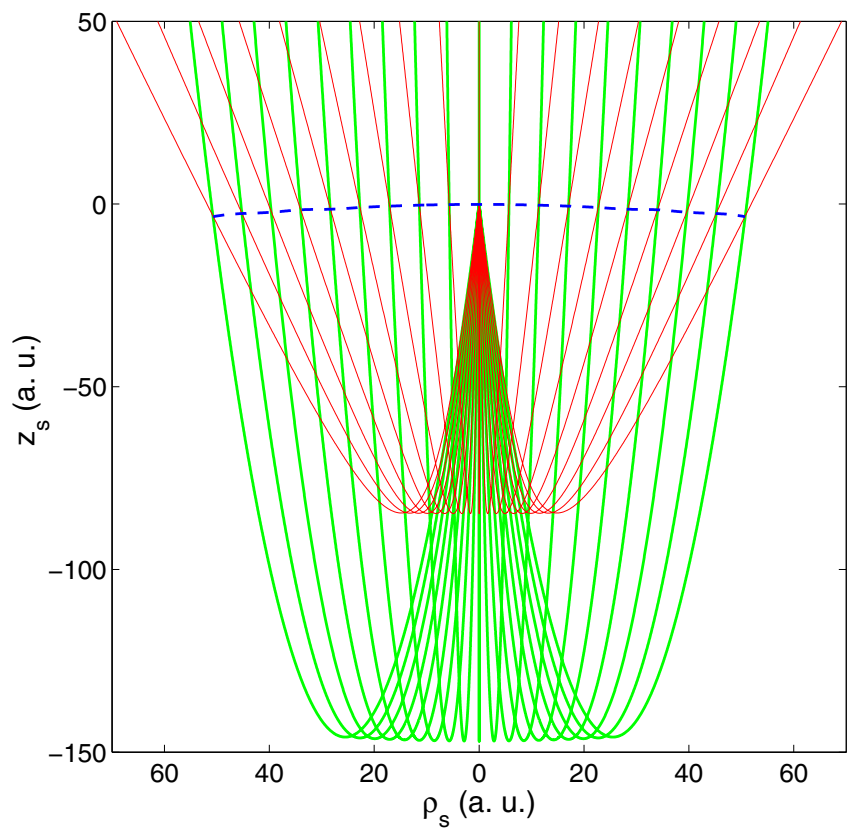

FIG. 7. (Color online) Dynamical origin of the equal oscillation amplitudes in Fig. 6. Thin (red) trajectories are for a detached electron near a repulsive center, and thick (green) trajectories are for a static electric field $F=\frac{\alpha}{d^{2}}$. Here $d=200 a_{0}, \alpha=1$, and $E=0.1 \mathrm{eV}$. Note that the two trajectories ejected with the same angle close to $\pi$ in the two different systems first move downward and then return, and they finally cross on the dashed line, which is perpendicular to the $z$ axis.

oscillations in the total cross section for photodetachment near a repulsive center. These studies were motivated by experiments on photodetachment of a dicarboxylate dianion [2], ${ }^{-} \mathrm{O}_{2} \mathrm{C}-\left(\mathrm{CH}_{2}\right)_{m}-\mathrm{CO}_{2}^{-}$(with $3 \leqslant m \leqslant 11$ ), where the intramolecular Coulomb repulsive force plays a significant role.

Based on closed-orbit theory $[6,16]$, we derived a formula in Eq. (20) for the photodetachment cross section above the zero-field threshold. The formula was extended to a uniform one in Eq. (25) applicable for energy above and below the zero-field threshold. The accuracy of the present results was shown to be excellent by comparing with both direct numerical integration over the differential cross section [1] and quantum calculations based on the exact Coulomb Green's function.

We discussed several features in the total photodetachment cross section near a repulsive center, including the oscillatory structure, the shift of the photoelectron threshold, and the effect of wave-source parity on the phase of the oscillation. We also demonstrated that the oscillation amplitude in the cross section near a repulsive center is the same as that in a static electric field if the static electric-field strength $F=\frac{\alpha}{d^{2}}$, where $d$ measures the distance between the negative ion and the repulsive center and $\alpha$ is the negative-charge number. This peculiar relationship is rooted in dynamics and is independent of the negative ion.

\section{ACKNOWLEDGMENTS}

B.C.Y. appreciates helpful discussions with C. Bracher. B.C.Y. and M.L.D. acknowledge support from National Science Foundation of China Grants No. 11074260 and No.
11121403. J.B.D. acknowledges support from NSF Grant No. 1068344.

\section{APPENDIX A: CONVERTING EQS. (12) AND (13) TO EQS. (14) AND (15)}

For convenience, we use $\Phi$ to denote the wave source $D \psi_{i}$, and $\psi$ in the following formulas represents the direct-outgoing wave or the returning wave. Substituting the inhomogeneous Schrödinger equation in Eq. (1) into Eqs. (12) and (13), we first write the integrand in Eqs. (12) and (13) as

$$
\operatorname{Im}\left(\Phi^{*} \psi\right)=-\frac{1}{2}\left(k^{2}-2 V\right) \operatorname{Im}\left(\psi^{*} \psi_{\text {out }}\right)-\frac{1}{2} \operatorname{Im}\left(\psi^{*} \nabla_{s}^{2} \psi_{\text {out }}\right)
$$

which can be transformed into

$$
\begin{aligned}
\operatorname{Im}\left(\Phi^{*} \psi\right)= & -\frac{1}{2} \operatorname{Im}\left[\psi_{\text {out }}\left(\nabla_{s}^{2}+k^{2}-2 V\right) \psi^{*}\right] \\
& +\frac{1}{2} \nabla_{s} \operatorname{Im}\left(\psi_{\text {out }} \nabla_{s} \psi^{*}-\psi^{*} \nabla_{s} \psi_{\text {out }}\right)
\end{aligned}
$$

after applying the following equation:

$$
\nabla_{s}\left(\psi_{\text {out }} \nabla_{s} \psi^{*}-\psi^{*} \nabla_{s} \psi_{\text {out }}\right)=\psi_{\text {out }} \nabla_{s}^{2} \psi^{*}-\psi^{*} \nabla_{s}^{2} \psi_{\text {out }} .
$$

Integrating both sides of Eq. (A2) and using the divergence theorem on the right-hand side, we have

$$
\begin{aligned}
\operatorname{Im}\left\langle D \psi_{i} \mid \psi\right\rangle= & -\frac{1}{2} \operatorname{Im} \int\left[\psi_{\text {out }}\left(\nabla_{s}^{2}+k^{2}-2 V\right) \psi^{*}\right] d^{3} \mathbf{r}_{s} \\
& +\frac{1}{2} \operatorname{Im} \int\left(\psi_{\text {out }} \nabla_{r_{s}} \psi^{*}-\psi^{*} \nabla_{r_{s}} \psi_{\text {out }}\right) d s_{r_{s} \rightarrow \infty}
\end{aligned}
$$

Equation (14) is obtained by using the inhomogeneous Schrödinger equation in Eq. (1) again in the above equation. If $\psi$ is the returning wave, the first term in Eq. (A4) vanishes and only the second integration survives, giving Eq. (15).

\section{APPENDIX B: THE DERIVATION OF EQS. (17) AND (18)}

Assuming $r_{s}$ is sufficiently large, the two integrands in Eqs. (17) and (18) can be written out using Eqs. (4) and (9) as

$$
\psi_{\text {out }} \nabla_{r_{s}} \psi_{\text {ret }}^{s^{*}}=-i k \mathcal{C}_{l m} \cos \theta_{s} Y_{l m}\left(\theta_{s}, \varphi\right) \frac{e^{i k r_{s}}}{r_{s}} e^{-i k r_{s} \cos \theta_{s}},
$$

and

$$
\begin{aligned}
\psi_{\mathrm{ret}}^{s^{*}} \nabla_{r_{s}} \psi_{\mathrm{out}}= & i k \mathcal{C}_{l m} Y_{l m}\left(\theta_{s}, \varphi\right) \frac{e^{i k r_{s}}}{r_{s}} e^{-i k r_{s} \cos \theta_{s}} \\
& -\mathcal{C}_{l m} Y_{l m}\left(\theta_{s}, \varphi\right) \frac{e^{i k r_{s}}}{r_{s}^{2}} e^{-i k r_{s} \cos \theta_{s}},
\end{aligned}
$$

where $\mathcal{C}_{l m}$ is defined as

$$
\mathcal{C}_{l m}=|C(k)|^{2} Y_{l m}(\pi, \phi) \frac{A}{R_{s}} e^{-i\left(S-\frac{\pi}{2}\right)} .
$$

Using the relationship in spherical harmonics

$$
\cos \theta_{s} Y_{l m}=b_{l m} Y_{l+1, m}+b_{l-1, m} Y_{l-1, m},
$$

where

$$
b_{l m}=\sqrt{\frac{(l+1)^{2}-m^{2}}{(2 l+1)(2 l+3)}},
$$


the integration of Eq. (B1) can be written as

$\int \psi_{\mathrm{out}} \nabla_{r_{s}} \psi_{\mathrm{ret}}^{s^{*}} d s_{r_{s} \rightarrow \infty}=-i k \mathcal{C}_{l m}\left(b_{l m} \mathcal{D}_{l+1, m}+b_{l-1, m} \mathcal{D}_{l-1, m}\right)$,

where $\mathcal{D}_{l m}$ is defined as

$$
\mathcal{D}_{l m}=\int_{r_{s} \rightarrow \infty} Y_{l m}\left(\theta_{s}, \varphi\right) e^{i k r_{s}} e^{-i k r_{s} \cos \theta_{s}} r_{s} \sin \theta_{s} d \theta_{s} d \varphi .
$$

It can be decomposed as

$$
\begin{aligned}
\mathcal{D}_{l m}= & \sum_{l^{\prime}=0}^{\infty} \sqrt{4 \pi\left(2 l^{\prime}+1\right)}(-i)^{l^{\prime}} j_{l^{\prime}}\left(k r_{s}\right) r_{s} e^{i k r_{s}} \\
& \times \int Y_{l m}\left(\theta_{s}, \varphi\right) Y_{l^{\prime} 0}^{*}\left(\theta_{s}\right) \sin \theta_{s} d \theta_{s} d \varphi,
\end{aligned}
$$

where $j_{l^{\prime}}\left(k r_{s}\right)$ is a spherical Bessel function. Because the spherical harmonics are orthogonal and normalized, we then have

$$
\mathcal{D}_{l m}=\sqrt{4 \pi(2 l+1)}(-i)^{l} j_{l}\left(k r_{s}\right) r_{s} e^{i k r_{s}} \delta_{m 0} .
$$

Using the following asymptotic formula,

$$
j_{l}\left(k r_{s}\right) \rightarrow \frac{1}{k r_{s}} \sin \left(k r_{s}-\frac{l \pi}{2}\right), r_{s} \rightarrow \infty,
$$

we finally have

$$
\mathcal{D}_{l m}=\frac{i}{2 k} \sqrt{4 \pi(2 l+1)}\left[1-e^{i\left(2 k r_{s}-l \pi\right)}\right] \delta_{m 0},
$$

when the radius $r_{s}$ is large.

The result in Eq. (17) is obtained by substituting $\mathcal{D}_{l m}$ into Eq. (B6). The integration of the second term in Eq. (B2) vanishes for large $r_{s}$ as a result of the asymptotic behavior of the spherical Bessel function at infinity in Eq. (B10), and the first term can be written out as in Eq. (18) using $\mathcal{D}_{l m}$ in Eq. (B11).

\section{APPENDIX C: MODULATION FUNCTION IN A HOMOGENEOUS ELECTRIC FIELD}

As we mentioned, the semiclassical modulation function $\mathcal{H}^{F}(S)$ in a static electric field is

$$
\mathcal{H}^{F}=\left[1-(-1)^{l}(2 l+1) \frac{1}{3 S} \cos (S) \delta_{m 0}\right],
$$

which can be shown using steps analogous to those that led to Eq. (21).

Using the exact Green's functions, Bracher et al. have shown that both the total cross section without any field and that in a uniform static electric field can be, respectively, obtained as $[9,10]$

$$
\sigma_{0}=\frac{\left|\lambda_{l m}\right|^{2}}{4 \pi^{2}} k^{2 l+1}
$$

and

$$
\sigma=\frac{\left|\lambda_{l m}\right|^{2}}{2 \pi} \sum_{j=|m|}^{l} \frac{k^{2 l+1}}{(2 \sqrt{-\zeta})^{2 l+1}} 2^{j}(2 j+1) ! ! T_{j l m}^{2} \mathrm{Qi}_{3 j-2 l+1}(\zeta)
$$

for an idealized multipole point source

$$
D \psi_{i}=\lambda_{l m} \delta_{l m}(\mathbf{r}-\mathbf{d}),
$$

where $\lambda_{l m}$ is a source strength and $\delta_{l m}(\mathbf{r}-\mathbf{d})$ is a multipole $\delta$ function with angular momentum $(l, m)$ relative to the source point at $\mathbf{d}$ (see $[9,10]$ for details). $T_{j l m}$ and $\mathrm{Qi}_{v}(\zeta)$ in Eq. (C3) are defined as

$$
\begin{gathered}
T_{j l m}=\frac{1}{(l-j) !} \sqrt{\frac{(2 l+1)(l+m) !(l-m) !}{(2 j+1)(j+m) !(j-m) !}} \\
\mathrm{Qi}_{\nu}(\zeta)=\lim _{z \rightarrow 0}\left(-\frac{1}{2 z} \frac{\partial}{\partial z}\right)^{v} \operatorname{Ai}(\zeta-z) \operatorname{Ai}(\zeta+z), \\
\mathrm{Qi}_{-v}(\zeta)=\lim _{z \rightarrow 0} \frac{\partial^{v}}{\partial z^{v}}[\operatorname{Ai}(\zeta-z)]^{2}, \quad v \geqslant 0 .
\end{gathered}
$$

Although the cross section in $[9,10]$ is defined slightly differently from ours, the modulation function is independent of the specific definition for the cross sections because it is the ratio of the cross section in the static electric field to the zero-field cross section. Therefore, the exact form of the modulation function $\mathcal{H}^{F}$ can be obtained as

$$
\mathcal{H}^{F}(\zeta)=\sum_{j=|m|}^{l} \frac{2 \pi}{(2 \sqrt{-\zeta})^{2 l+1}} 2^{j}(2 j+1) ! ! T_{j l m}^{2} \mathrm{Qi}_{3 j-2 l+1}(\zeta)
$$

by using Eqs. (C2) and (C3) in Eq. (20).

\section{APPENDIX D: TOTAL CROSS-SECTION FORMULAS BASED ON EXACT COULOMB GREEN'S FUNCTION}

Here it is most convenient to use the coordinates relative to the repulsive charge center. The exact Coulomb Green's function can be written as [24-27]

$$
\begin{aligned}
G(\mathbf{r}, \mathbf{d}, \epsilon)= & \frac{\Gamma(1-i \eta)}{2 \pi|\mathbf{r}-\mathbf{d}|}\left[\mathcal{W}_{i \eta, 1 / 2}^{\prime}(\xi) \mathcal{M}_{i \eta, 1 / 2}(\zeta)\right. \\
& \left.-\mathcal{W}_{i \eta, 1 / 2}(\xi) \mathcal{M}_{i \eta, 1 / 2}^{\prime}(\zeta)\right],
\end{aligned}
$$

which satisfies the inhomogeneous Schrödinger equation with a delta source:

$$
\left(\frac{1}{2} \nabla^{2}-\frac{\alpha}{r}+\epsilon\right) G(\mathbf{r}, \mathbf{d}, \epsilon)=\delta(\mathbf{r}-\mathbf{d})
$$

where the total energy $\epsilon=E+\alpha / d, \eta=-\alpha / \kappa$ with $\kappa=$ $\sqrt{2 \epsilon}, \xi=-i \kappa(r+d+|\mathbf{r}-\mathbf{d}|)$, and $\zeta=-i \kappa(r+d-\mid \mathbf{r}-$ $\mathbf{d} \mid) . \Gamma(1-i \eta)$ is a gamma function [28]. $\mathcal{M}_{i \eta, 1 / 2}(\zeta)$ and $\mathcal{W}_{i \eta, 1 / 2}(\xi)$ are, respectively, the Whittaker functions of the first kind and the second kind [28].

Following the method developed by Bracher et al. $[9,10]$, the total cross section for the multipole source in Eq. (C4) is given by the following equation:

$$
\sigma=-2\left|\lambda_{l m}\right|^{2} \operatorname{Im}\left[\lim _{\mathbf{r} \rightarrow \mathbf{d}} K_{l m}^{*}\left(\frac{\partial}{\partial \mathbf{r}}\right) G_{l m}(\mathbf{r}, \mathbf{d} ; \epsilon)\right],
$$


where $G_{l m}(\mathbf{r}, \mathbf{d} ; \epsilon)$ is a multipole Green's function and can be obtained from the usual Green's function as

$$
G_{l m}(\mathbf{r}, \mathbf{d} ; \epsilon)=K_{l m}\left(\frac{\partial}{\partial \mathbf{d}}\right) G(\mathbf{r}, \mathbf{d} ; \epsilon) .
$$

The spherical tensor gradient operator $K_{l m}\left(\frac{\partial}{\partial \mathbf{r}}\right)$ in Eqs. (D3) and (D4) is defined by replacing the Cartesian components of $\mathbf{r}=(x, y, z)$ in the argument of the harmonic polynomial $K_{l m}(\mathbf{r})=r^{l} Y_{l m}(\theta, \varphi)$ by the Cartesian components of $\nabla=$ $\left(\frac{\partial}{\partial x}, \frac{\partial}{\partial y}, \frac{\partial}{\partial z}\right)[29,30]$.

According to the definition in Eq. (22), the reduced cross section can be written out from Eqs. (D3) and (C2) as

$$
\tilde{\sigma}=-8 \pi^{2} \operatorname{Im}\left[\lim _{\mathbf{r} \rightarrow \mathbf{d}} K_{l m}^{*}\left(\frac{\partial}{\partial \mathbf{r}}\right) G_{l m}(\mathbf{r}, \mathbf{d} ; \epsilon)\right] .
$$

Substituting the exact Coulomb Green's function in Eq. (D1) into Eq. (D5), the closed-form expression of the reduced cross section for an $s$-wave source is obtained as

$$
\begin{aligned}
\tilde{\sigma}= & \kappa e^{\pi \eta}|\Gamma(1-i \eta)|^{2}\left[\mathcal{M}_{i \eta, 1 / 2}^{\prime 2}(-2 i \kappa d)\right. \\
& \left.-\frac{1}{4}\left(1+\frac{2 \eta}{\kappa d}\right) \mathcal{M}_{i \eta, 1 / 2}^{2}(-2 i \kappa d)\right],
\end{aligned}
$$

and the closed-form expression for a $p_{z}$-wave source is written out as

$$
\begin{aligned}
\tilde{\sigma}= & \kappa^{3} e^{\pi \eta}|\Gamma(1-i \eta)|^{2}\left\{\left(1+\frac{2 \eta}{\kappa d}\right) \mathcal{M}_{i \eta, 1 / 2}^{\prime 2}(-2 i \kappa d)\right. \\
& -\frac{2 i \eta}{\kappa^{2} d^{2}} \mathcal{M}_{i \eta, 1 / 2}(-2 i \kappa d) \mathcal{M}_{i \eta, 1 / 2}^{\prime}(-2 i \kappa d) \\
& \left.-\frac{1}{4}\left[\left(1+\frac{2 \eta}{\kappa d}\right)^{2}+\frac{2 \eta}{\kappa^{3} d^{3}}\right] \mathcal{M}_{i \eta, 1 / 2}^{2}(-2 i \kappa d)\right\}
\end{aligned}
$$

in terms of the Whittaker function of the first kind.
[1] B. C. Yang, J. B. Delos, and M. L. Du, Phys. Rev. A 88, 023409 (2013).

[2] X. P. Xing, X. B. Wang, and L. S. Wang, Phys. Rev. Lett. 101, 083003 (2008); J. Chem. Phys. 130, 074301 (2009).

[3] I. I. Fabrikant, Zh. Eksp. Teor. Fiz. 79, 2070 (1980) [Sov. Phys.— JETP 52, 1045 (1980)]; Zh. Eksp. Teor. Fiz. 83, 1675 (1982) [Sov. Phys._JETP 56, 967 (1982)].

[4] N. Y. Du, I. I. Fabrikant, and A. F. Starace, Phys. Rev. A 48, 2968 (1993); I. I. Fabrikant, J. Phys. B 27, 4545 (1994).

[5] M. L. Du and J. B. Delos, Phys. Rev. A 38, 5609 (1988); Phys. Lett. A 134, 476 (1989).

[6] M. L. Du, Phys. Rev. A 70, 055402 (2004); Eur. Phys. J. D 38, 533 (2006).

[7] A. R. P. Rau and H.-Y. Wong, Phys. Rev. A 37, 632 (1988); H.-Y. Wong, A. R. P. Rau, and C. H. Greene, ibid. 37, 2393 (1988); C. H. Greene, ibid. 36, 4236 (1987).

[8] N. L. Manakov, M. V. Frolov, A. F. Starace, and I. I. Fabrikant, J. Phys. B 33, R141 (2000).

[9] C. Bracher, T. Kramer, and M. Kleber, Phys. Rev. A 67, 043601 (2003).

[10] C. Bracher, Ph.D. thesis, Technische Universität München, 1999.

[11] C. Bracher and J. B. Delos, Phys. Rev. Lett. 96, 100404 (2006); C. Bracher, T. Kramer, and J. B. Delos, Phys. Rev. A 73, 062114 (2006); C. Bracher and A. Gonzalez, ibid. 86, 022715 (2012).

[12] H. C. Bryant et al., Phys. Rev. Lett. 58, 2412 (1987); J. E. Stewart et al., Phys. Rev. A 38, 5628 (1988); P. G. Harris et al., ibid. 41, 5968 (1990).

[13] N. D. Gibson, B. J. Davies, and D. J. Larson, Phys. Rev. A 47, 1946 (1993); 48, 310 (1993); N. D. Gibson, M. D. Gasda, K. A. Moore, D. A. Zawistowski, and C. W. Walter, ibid. 64, 061403(R) (2001).

[14] W. A. M. Blumberg, R. M. Jopson, and D. J. Larson, Phys. Rev. Lett. 40, 1320 (1978); J. N. Yukich, T. Kramer, and C. Bracher, Phys. Rev. A 68, 033412 (2003).
[15] R. I. Rabinovich, Zh. Eksp. Teor. Fiz. 88, 1718 (1985) [Sov. Phys._JETP 61, 1022 (1985)].

[16] M. L. Du and J. B. Delos, Phys. Rev. Lett. 58, 1731 (1987); Phys. Rev. A 38, 1896 (1988); 38, 1913 (1988).

[17] M. L. Du, Phys. Rev. A 40, 4983 (1989).

[18] F. W. J. Olver, D. W. Lozier, R. F. Boisvert, and C. W. Clark, NIST Handbook of Mathematical Functions (Cambridge University Press, New York, 2010).

[19] E. P. Wigner, Phys. Rev. 73, 1002 (1948).

[20] M. Scheer, R. C. Bilodeau, J. Thøgersen, and H. K. Haugen, Phys. Rev. A 57, R1493 (1998); M. Scheer, C. A. Brodie, R. C. Bilodeau, and H. K. Haugen, ibid. 58, 2051 (1998)

[21] J. A. Shaw, J. B. Delos, M. Courtney, and D. Kleppner, Phys. Rev. A 52, 3695 (1995).

[22] L. S. Wang, C. F. Ding, X. B. Wang, and J. B. Nicholas, Phys. Rev. Lett. 81, 2667 (1998); X. B. Wang, C. F. Ding, and L. S. Wang, Chem. Phys. Lett. 307, 391 (1999).

[23] M. L. Du, arXiv:0912.5257.

[24] L. Hostler and R. H. Pratt, Phys. Rev. Lett. 10, 469 (1963); L. Hostler, J. Math. Phys. 5, 591 (1964); 5, 1235 (1964).

[25] J. Schwinger, J. Math. Phys. 5, 1606 (1964).

[26] S. M. Blinder, J. Math. Phys. 22, 306 (1981); Int. J. Quantum Chem. 26, 293 (1984).

[27] V. Kanellopoulos, M. Kleber, and T. Kramer, Phys. Rev. A 80, 012101 (2009).

[28] Handbook of Mathematical Functions with Formulas, Graphs, and Mathematical Tables, edited by M. Abramawitz and I. A. Stegun (Dover, New York, 1965).

[29] B. F. Bayman, J. Math. Phys. 19, 2558 (1978); E. G. P. Rowe, ibid. 19, 1962 (1978).

[30] E. J. Weniger and E. O. Steinborn, J. Math. Phys. 24, 2553 (1983); E. J. Weniger, Collect. Czech. Chem. Commun. 70, 1225 (2005). 